\title{
The Concepts of Tightness for Fuzzy Set Valued Random Variables
}

\author{
Yun Kyong Kim \\ Department of Information \& Communication Engineering, Dongshin University, Daehodong 252, Naju, \\ Chonnam, 520-714, Korea
}

\begin{abstract}
In this paper, we introduce several concepts of tightness for a sequence of random variables taking values in the space of normal and upper-semicontinuous fuzzy sets with compact support in $R^{p}$ and give some characterizations of their concepts. Also, counter-examples for the relationships between the concepts of tightness are given.
\end{abstract}

Key Words : Fuzzy sets, Random Sets, Fuzzy random variables, Tightness.

\section{Introduction}

The theory of fuzzy sets introduced by Zadeh has been extensively studied and applied in statistics and probability areas in recent years because of its usefulness in several applied fields. The central concept of them is a fuzzy random variable, which was introduced in order to give a way of handling random experiments whose outcomes are not numbers but are expressed in ambiguous linguistic terms due to the subjectivity and imprecision of human knowledge. (See Klement et al. [16], Li et al. [17], Puri and Ralescu [20])

The notion of tightness of probability measures plays an important role both in the theory of weak convergence and in its applications. Prokhorov [19] gave the theory of weak convergence of probability measures on complete separable metric spaces. As one knows, his work made a great contribution to developments of modern probability theory. The relationships between weak convergence and tightness of probability measures can be also found in Billingsley [1].

The concept of tightness for fuzzy random variables was firstly introduced by Inoue [9] in order to obtain strong law of large numbers for fuzzy random variables. Recently, Joo and Kim [11], Joo et al.[12, 13] introduced a new concept of tightness for fuzzy random variables in order to study convergence in distribution of fuzzy random variables. By the way, it is possible to define several concepts of tightness for fuzzy random variables since there are many useful metrics defined on the space of fuzzy sets.

The purpose of this paper is to introduce several concepts of tightness for fuzzy random variables and give some characterizations of their concepts. Also, counter-examples for the relationships between the concepts of tightness are given.

\section{Preliminaries}

Let $\mathcal{K}\left(R^{p}\right)$ denote the family of non-empty compact subsets of the Euclidean space $R^{p}$. Then the space $\mathcal{K}\left(R^{p}\right)$ is metrizable by the Hausdorff metric $h$ defined by

$$
h(A, B)=\max \left\{\sup _{a \in A} \inf _{b \in B}|a-b|, \sup _{b \in B} \inf _{a \in A}|a-b|\right\} .
$$

A norm of $A \in \mathcal{K}\left(R^{p}\right)$ is defined by

$$
\|A\|=h(A,\{0\})=\sup _{a \in A}|a| .
$$

It is well-known that $\mathcal{K}\left(R^{p}\right)$ is complete and separable with respect to the Hausdorff metric $h$ (see Debreu [3]).

Lemma 2.1. (Diamond and Kloeden [5]). Let $\mathcal{A}$ be a subset of $\left(\mathcal{K}\left(R^{p}\right), h\right)$. Then $\mathcal{A}$ is relatively compact if and only if $\sup _{A \in \mathcal{A}}\|A\|<\infty$.

Let $\mathcal{F}\left(R^{p}\right)$ denote the family of all fuzzy sets $u: R^{p} \rightarrow$ $[0,1]$ with the following properties;

(i) $u$ is normal, i.e., there exists $x \in R^{p}$ such that $u(x)=$ 1 ;

(ii) $u$ is upper semicontinuous;

(iii) $\operatorname{supp} u=c l\left\{x \in R^{p}: u(x)>0\right\}$ is compact, where $c l$ denotes the closure.

Manuscript received Feb. 11. 2008; revised May. 15. 2008.

Corresponding Author : Yun Kyong Kim, ykkim@dsu.ac.kr

The research was supported by the Korea Research Grant funded by the Korean Government (KRF-2007-313-C00075). 
For a fuzzy subset $u$ of $R^{p}$, the $\alpha$-level set of $u$ is defined by

$$
L_{\alpha} u=\left\{\begin{array}{lcc}
\{x: u(x) \geq \alpha\} & \text { if } & 0<\alpha \leq 1, \\
\operatorname{supp} u & \text { if } & \alpha=0 .
\end{array}\right.
$$

Then it follows immediately that $u \in \mathcal{F}\left(R^{p}\right)$ if and only if $L_{\alpha} \in \mathcal{K}\left(R^{p}\right)$ for each $\alpha \in[0,1]$.

Theorem 2.2. For each $u \in \mathcal{F}\left(R^{p}\right)$, we define

$$
f_{u}:[0,1] \rightarrow\left(\mathcal{K}\left(R^{p}\right), h\right), f_{u}(\alpha)=L_{\alpha} u
$$

Then the followings are satisfied;

1. $f_{u}$ is non-increasing w.r.t. set inclusion.

2. $f_{u}$ is left-continuous in $(0,1]$ and $f_{u}$ is rightcontinuous at 0 .

3. $f_{u}$ has right-limit at each $\alpha \in(0,1]$.

Conversely, if $g:[0,1] \rightarrow\left(\mathcal{K}\left(R^{p}\right), h\right)$ is a function satisfying (1) - (3), then there exists a unique $u \in \mathcal{F}\left(R^{p}\right)$ such that $g(\alpha)=L_{\alpha} u$ for all $\alpha$.

$u$ is called level-continuous if $f_{u}$ is continuous. If the right limit of $f_{u}$ at $\alpha$ is denoted by $L_{\alpha^{+}} u$, then

$$
L_{\alpha^{+}} u=c l\left\{x \in R^{p}: u(x)>\alpha\right\} .
$$

For details, see Joo and Kim [10].

Now we define the uniform metric $d_{\infty}$ on $\mathcal{F}\left(R^{p}\right)$ as usual;

$$
d_{\infty}(u, v)=\sup _{0 \leq \alpha \leq 1} h\left(L_{\alpha} u, L_{\alpha} v\right) .
$$

Also, the norm of $u$ is defined as

$$
\|u\|=d_{\infty}(u, \tilde{0})=\left\|L_{0} u\right\|=\sup _{x \in L_{0} u}|x|,
$$

where $\tilde{0}$ denotes the indicator function of $\{0\}$.

It is well-known that $\left(\mathcal{F}\left(R^{p}\right), d_{\infty}\right)$ is complete but is not separable (see Klement et al. [16]).

Another metrics on $\mathcal{F}\left(R^{p}\right)$ can be defined as follows;

$$
\begin{aligned}
d_{r}(u, v) & =\left(\int_{0}^{1} h\left(L_{\alpha} u, L_{\alpha} v\right)^{r} d \alpha\right)^{1 / r}, 1 \leq r<\infty \\
D(u, v) & =h(\operatorname{send}(u), \operatorname{send}(v)),
\end{aligned}
$$

where $\operatorname{send}(u)=\left\{(x, \alpha) \in R^{p} \times[0,1]: x \in L_{0} u\right.$ and $\mathrm{u}(\mathrm{x}) \geq$ $\alpha\}$ is the sendograph of $u$.

The metrics $d_{r}$ and $D$ are called the $L_{r}$-metric and sendograph metric, respectively. Relations for convergence of fuzzy sets w.r.t. these metrics can be found in Diamond and Kloeden [5]. Another useful metric was introduced by Joo and Kim [10] as follows.
Definition 2.3. Let $T$ denote the class of strictly increasing, continuous mapping of $[0,1]$ onto itself. For $u, v \in \mathcal{F}\left(R^{p}\right)$, we define

$$
\begin{aligned}
d_{s}(u, v)= & \inf \{\varepsilon>0: \text { there exists a } t \in T \text { such that } \\
& \left.\sup _{0 \leq \alpha \leq 1}|t(\alpha)-\alpha| \leq \varepsilon \text { and } d_{\infty}(u, t(v)) \leq \varepsilon\right\}
\end{aligned}
$$

where $t(v)$ denotes the composition of $v$ and $t$.

The metric $d_{s}$ will be called the Haudorff-Skorokhod metric. It is well-known that $\left(\mathcal{F}\left(R^{p}\right), d_{s}\right)$ is separable and topologically complete.

\section{Main Results}

Throughout this paper, let $(\Omega, \mathcal{B}, \mathcal{P})$ be a probability space. A set-valued function $X: \Omega \rightarrow \mathcal{K}\left(R^{p}\right)$ is called measurable if for each closed subset $B$ of $R^{p}$,

$$
X^{-w}(B)=\{\omega: X(\omega) \cap B \neq \emptyset\} \in \mathcal{B} .
$$

It is well-known that the measurablility of $X$ is equivalent to the measurability of $X$ considered as a map from $\Omega$ into the metric space $\mathcal{K}\left(R^{p}\right)$ endowed with the Hausdorff metric $h$. A set valued function $X: \Omega \rightarrow \mathcal{K}\left(R^{p}\right)$ is called a random set if it is measurable.

A fuzzy set valued function $\tilde{X}: \Omega \rightarrow \mathcal{F}\left(R^{p}\right)$ is called measurable if for each $\alpha \in[0,1], L_{\alpha} \tilde{X}$ is measurable set valued function.

A fuzzy set valued function is called a fuzzy random variable(or fuzzy random set) if it is measurable.

It is well-known that $\tilde{X}$ is a fuzzy random variable if and only if $\tilde{X}: \Omega \rightarrow\left(\mathcal{F}\left(R^{p}\right), d_{s}\right)$ is measurable (For details, see Colubi et al. [2], Kim [15]).

Definition 3.1. Let $\left\{\tilde{X}_{n}, n=1,2, \ldots\right\}$ be a sequence of fuzzy random variables.

(1). $\left\{\tilde{X}_{n}\right\}$ is said to be uniformly level-wise tight (ULT) if for each $\varepsilon>0$, there exists a compact subset $\mathcal{A}$ of $\left(\mathcal{K}\left(R^{p}\right), h\right)$ such that

$$
P\left(L_{\alpha} \tilde{X}_{n} \notin \mathcal{A}\right)<\varepsilon \text { for all nand all } \alpha \in[0,1] .
$$

(2). $\left\{\tilde{X}_{n}\right\}$ is said to be tight $(\mathbf{T})$ if for each $\varepsilon>0$, there exists a compact subset $\mathcal{U}$ of $\left(\mathcal{F}\left(R^{p}\right), d_{s}\right)$ such that

$$
P\left(\tilde{X}_{n} \notin \mathcal{U}\right)<\varepsilon \text { for all } n \text {. }
$$

If we can choose $\mathcal{U}$ to be compact with respect to the metric $d_{\infty}, d_{r}$ or the sendograph metric $D$, then $\left\{\tilde{X}_{n}\right\}$ will be called strongly tight $(\mathbf{S T}), L_{r}$-tight $\left(\mathbf{L}_{\mathbf{r}} \mathbf{T}\right)$ or graphically tight $(\mathbf{G T})$, respectively. 
Note that the concept of tightness introduced by Inoue [9] is uniformly level-wise tight in our sense. Characterizations of compact subsets of $\left(\mathcal{F}\left(R^{p}\right), d_{s}\right)$ and tightness of fuzzy random variables were obtained in $[11,12]$. To review the main results, we need some notations. For $u \in \mathcal{F}\left(R^{p}\right)$ and $0<\delta<1$, we define

$$
\phi_{s}(u, \delta)=\inf _{\left\{\alpha_{i}\right\}} \max _{1 \leq i \leq r} h\left(L_{\alpha_{i-1}^{+}} u, L_{\alpha_{i}} u\right)
$$

where the infimum is taken over all partitions $0=\alpha_{0}<$ $\alpha_{1}<\cdots<\alpha_{r}=1$ of $[0,1]$ satisfying $\min _{1 \leq i \leq r}\left(\alpha_{i}-\alpha_{i-1}\right)>$ $\delta$.

Theorem 3.2. (Joo and $\operatorname{Kim}[11]) .\left\{\tilde{X}_{n}\right\}$ is tight if and only if

(3.1) For each $\varepsilon>0$, there exists a $\lambda>0$ such that

$$
P\left\{\omega:\left\|\tilde{X}_{n}(\omega)\right\|>\lambda\right\}<\varepsilon \text { for all } n .
$$

(3.2) For each $\varepsilon>0$ and $\eta>0$, there exists $a \delta \in(0,1)$ such that

$$
P\left\{\omega: \phi_{s}\left(\tilde{X}_{n}(\omega), \delta\right) \geq \eta\right\}<\varepsilon \text { for all } n .
$$

Note that the above condition (3.1) is equivalent to that $\left\{\left\|\tilde{X}_{n}\right\|\right\}$ is tight in the sense of real valued random variables. In fact, this is equivalent to ULT of $\left\{\tilde{X}_{n}\right\}$.

Theorem 3.3. Let $\left\{\tilde{X}_{n}\right\}$ be a sequence of fuzzy random variables. Then the following statements are equivalent.

1. $\left\{\tilde{X}_{n}\right\}$ is uniformly level-wise tight.

2. The sequence $\left\{L_{0} \tilde{X}_{n}\right\}$ of random sets is tight.

3. The sequence $\left\{\left\|\tilde{X}_{n}\right\|\right\}$ of real valued random variables is tight.

Proof. $(1) \Rightarrow(2)$ : It is trivial from Definition 3.1.

$(2) \Rightarrow(3)$ : Let $\left\{L_{0} \tilde{X}_{n}\right\}$ be tight. Then for each $\varepsilon>0$, there exists a compact subset $\mathcal{A}$ of $\left(\mathcal{K}\left(R^{p}\right), h\right)$ such that

$$
P\left(L_{0} \tilde{X}_{n} \notin \mathcal{A}\right)<\varepsilon \text { for all } n .
$$

By Lemma 2.1, there exists a $\lambda>0$ such that

$$
\|A\| \leq \lambda \text { for all } A \in \mathcal{A} .
$$

Since $\left\|\tilde{X}_{n}\right\|=\left\|L_{0} \tilde{X}_{n}\right\|$, we have that for all $n$,

$$
P\left\{\left\|\tilde{X}_{n}\right\|>\lambda\right\} \leq P\left(L_{0} \tilde{X}_{n} \notin \mathcal{A}\right)<\varepsilon .
$$

This implies that $\left\{\left\|\tilde{X}_{n}\right\|\right\}$ is tight.

$(3) \Rightarrow(1)$ : Suppose that $\left\{\left\|\tilde{X}_{n}\right\|\right\}$ is tight. Then for each $\varepsilon>0$, there exists $M>0$ such that for all $n$,

$$
P\left\{\left\|\tilde{X}_{n}\right\|>M\right\}<\varepsilon .
$$

Let $\mathcal{A}$ be the closure of $\left\{A \in \mathcal{K}\left(R^{p}\right):\|A\| \leq M\right\}$. By Lemma $2.1, \mathcal{A}$ is a compact subset of $\left(\mathcal{K}\left(R^{p}\right), h\right)$. Since $\left\|L_{\alpha} \tilde{X}_{n}\right\| \leq\left\|L_{0} \tilde{X}_{n}\right\|=\left\|\tilde{X}_{n}\right\|$, we have that for all $n$ and all $\alpha \in[0,1]$,

$$
\begin{aligned}
P\left(L_{\alpha} \tilde{X}_{n} \notin \mathcal{A}\right) & \leq P\left(\left\|L_{\alpha} \tilde{X}_{n}\right\|>M\right) \\
& \leq P\left\{\left\|\tilde{X}_{n}\right\|>M\right\}<\varepsilon .
\end{aligned}
$$

Now we intend to characterize graphical tightness for fuzzy random variables. To do this, we need characterizations of compact subsets of $\mathcal{F}\left(R^{p}\right)$ with respect to the sendograph metric $D$. This was obtained by Fan [6] in the case of $p=1$ and by Greco [7] in the general case. To give an explanation of their result, we denote

$$
\phi_{D}(u, \delta)=h\left(L_{0} u, L_{\delta} u\right)
$$

for $u \in \mathcal{F}\left(R^{p}\right)$ and $0<\delta \leq 1$.

Theorem 3.4 (Greco [7]). Let $\mathcal{U}$ be a subset of $\left(\mathcal{F}\left(R^{p}\right), D\right)$. Then $\mathcal{U}$ is relatively compact if and only if

(3.3) $\sup _{u \in \mathcal{U}}\|u\|<\infty$

(3.4) $\lim _{\delta \rightarrow 0} \sup _{u \in \mathcal{U}} \phi_{D}(u, \delta)=0$.

By using this result, we can obtain the following.

Theorem 3.5. Let $\left\{\tilde{X}_{n}\right\}$ be a sequence of fuzzy random variables. Then $\left\{\tilde{X}_{n}\right\}$ is graphically tight if and only if

(3.5) For each $\varepsilon>0$, there exists a $\lambda>0$ such that for all $n$,

$$
P\left\{\left\|\tilde{X}_{n}\right\|>\lambda\right\}<\varepsilon
$$

(3.6) For each $\varepsilon>0$ and $\eta>0$, there exists $a \delta \in(0,1)$ such that for all $n$,

$$
P\left(\phi_{D}\left(\tilde{X}_{n}, \delta\right) \geq \eta\right) \leq \varepsilon
$$

Proof. The proof will be proceeded by similar arguments as in Joo and Kim [11] and Joo et al. [13].

Suppose that $\left\{\tilde{X}_{n}\right\}$ is graphically tight. For given $\varepsilon>0$, there exists a compact subset $\mathcal{U}$ of $\left(\mathcal{F}\left(R^{p}\right), D\right)$ such that

$$
P\left(\tilde{X}_{n} \notin \mathcal{U}\right)<\varepsilon \text { for all } n \text {. }
$$

By (3.3) of Theorem 3.4, we have that

$$
\mathcal{U} \subset\{u:\|u\| \leq \lambda\} \text { for large enough } \lambda,
$$


which proves (3.5). Now for (3.6), let $\eta>0$ be given. Then by (3.4) of Theorem 3.4, there exists a $\delta>0$ such that

$$
\mathcal{U} \subset\left\{u: \phi_{D}(u, \delta)<\eta\right\}
$$

Therefore (3.6) follows immediately.

To prove the converse, suppose that (3.5) and (3.6) are valid. For given $\varepsilon>0$, we choose $\lambda>0$ so that for all $n$,

$$
P\left(\left\|\tilde{X}_{n}\right\|>\lambda\right\}<\varepsilon / 2 .
$$

And then for each positive integer $k$, we choose $\delta_{k}>0$ so that for all $n$,

$$
P\left(\phi_{D}\left(\tilde{X}_{n}, \delta_{k}\right) \geq 1 / k\right) \leq \varepsilon / 2^{k+1} .
$$

Let us denote

$$
\mathcal{U}_{0}=\{u:\|u\| \leq \lambda\}
$$

and for $k \geq 1$,

$$
\left.\left.\mathcal{U}_{k}=\left\{u: \phi_{D}\right) u, \delta_{k}\right)<1 / k\right\} .
$$

If $\mathcal{U}$ is the closure of $K=\cap_{k=0}^{\infty} \mathcal{U}_{k}$, then $\mathcal{U}$ is compact subset of $\left(\mathcal{F}\left(R^{p}\right), D\right)$ since $K$ satisfies (3.3) and (3.4) of Theorem 3.4. And then for all $n$,

$$
P\left(\tilde{X}_{n} \notin \mathcal{U}\right) \leq \sum_{k=0}^{\infty} P\left(\tilde{X}_{n} \notin \mathcal{U}_{k}\right)<\varepsilon .
$$

This implies that $\left\{\tilde{X}_{n}\right\}$ is graphically tight.

Turning our concern to characterize strong tightness for fuzzy random variables, we need to characterize compact subsets of $\left(\mathcal{F}\left(R^{p}\right), d_{\infty}\right)$. This was obtained by Diamond and Kloeden $[4,5]$ in the case of

$$
\begin{aligned}
F\left(R^{p}\right)=\{u \in & \mathcal{F}\left(R^{p}\right): u \text { is convex, i.e. } \\
u(\lambda x+(1-\lambda) y) \geq \min (u(x), u(y)) & \text { for } \left.x, y \in R^{p} \text { and } \lambda \in[0,1]\right\} .
\end{aligned}
$$

Joo et al. [13] established characterizations of compact subsets of $\left(\mathcal{F}_{C}\left(R^{p}\right), d_{\infty}\right)$, where

$$
\mathcal{F}_{C}\left(R^{p}\right)=\left\{u \in \mathcal{F}\left(R^{p}\right): u \text { is level-continuous }\right\},
$$

in order to study convergence in distribution for levelcontinuous fuzzy random variables. The result of [13] is that a subset $\mathcal{U}$ of $\left(\mathcal{F}_{C}\left(R^{p}\right), d_{\infty}\right)$ is relatively compact if and only if

(3.7) $\sup _{u \in \mathcal{U}}\|u\|<\infty$.

(3.8) $\lim _{\delta \rightarrow 0} \sup _{u \in \mathcal{U}} \phi_{\infty}(u, \delta)=0$,

where $\phi_{\infty}(u, \delta)=\sup _{|\alpha-\beta| \leq \delta} h\left(L_{\alpha} u, L_{\beta} u\right)$.

But in the space $\left(\mathcal{F}\left(R^{p}\right), d_{\infty}\right),(3.8)$ may not be satisfied even though $\mathcal{U}$ is a singleton subset. For example,

$$
u(x)=\left\{\begin{array}{llc}
1, & \text { if } & x=0 \\
1 / 2, & \text { if } & 0<|x| \leq 1 \\
0, & \text { elsewhere. } &
\end{array}\right.
$$

Then

$$
L_{\alpha} u= \begin{cases}\{x:|x| \leq 1\}, & \text { if } \quad 0 \leq \alpha \leq 1 / 2 \\ \{0\}, & \text { if } 1 / 2<\alpha \leq 1\end{cases}
$$

and so $\phi_{\infty}(u, \delta)=1$ for $0<\delta<1$.

This example shows that $\phi_{\infty}(u, \delta)$ must be modified in the case of $\mathcal{F}\left(R^{p}\right)$. This problem was solved by Greco and Moschen [8]. To explain the result, we adopt the following notations;

$$
\begin{gathered}
\phi^{\alpha}(u, \delta)=h\left(L_{\alpha} u, L_{\alpha-\delta} u\right), \\
\phi^{\alpha^{+}}(u, \delta)=h\left(L_{\alpha^{+}} u, L_{\alpha^{+}+\delta} u\right) .
\end{gathered}
$$

Theorem 3.6 (Greco and Moschen [8]). A subset $\mathcal{U}$ of $\left(\mathcal{F}\left(R^{p}\right), d_{\infty}\right)$ is relatively compact if and only if

(3.9) $\sup _{u \in \mathcal{U}}\|u\|<\infty$.

(3.10) For each $\alpha \in(0,1], \lim _{\delta \rightarrow 0} \sup _{u \in \mathcal{U}} \phi^{\alpha}(u, \delta)=0$.

(3.11) For each $\alpha \in[0,1), \lim _{\delta \rightarrow 0} \sup _{u \in \mathcal{U}} \phi^{\alpha^{+}}(u, \delta)=0$.

By using Theorem 3.6 and similar arguments as in the proof of Theorem 3.5, we can obtain the following result.

Theorem 3.7. Let $\left\{\tilde{X}_{n}\right\}$ be a sequence of fuzzy random variables. Then $\left\{\tilde{X}_{n}\right\}$ is strongly tight if and only if

1. $\left\{\left\|\tilde{X}_{n}\right\|\right\}$ is tight.

2. For each $\alpha \in(0,1], \varepsilon>0$ and $\eta>0$, there exists $a$ $\delta \in(0,1)$ such that for all $n$,

$$
P\left(\phi^{\alpha}\left(\tilde{X}_{n}, \delta\right) \geq \eta\right) \leq \varepsilon
$$

3. For each $\alpha \in[0,1), \varepsilon>0$ and $\eta>0$, there exists a $\delta \in(0,1)$ such that for all $n$,

$$
P\left(\phi^{\alpha^{+}}\left(\tilde{X}_{n}, \delta\right) \geq \eta\right) \leq \varepsilon
$$

The following theorem gives an useful sufficient condition for compactness in $\left(\mathcal{F}\left(R^{p}\right), d_{\infty}\right)$.

Theorem 3.8. Let $\mathcal{U}$ be a subset of $\left(\mathcal{F}\left(R^{p}\right), d_{\infty}\right)$. Then $\mathcal{U}$ is relatively compact if

(3.12) $\sup _{u \in \mathcal{U}}\|u\|<\infty$.

(3.13) $\lim _{\delta \rightarrow 0} \sup _{u \in \mathcal{U}} \phi_{\infty}(u, \delta)=0$ 
Proof. Suppose that (3.12) and (3.13) are valid. We show that $\mathcal{U}$ is sequentially compact, i.e., every sequence in $\mathcal{U}$ has a convergent subsequence.

Let $\left\{u_{n}\right\}$ be a sequence in $\mathcal{U}$. Then (3.12) implies that for each $\alpha \in[0,1],\left\{L_{\alpha} u_{n}\right\}$ is relatively compact subset of $\left(\mathcal{K}\left(R^{p}\right), h\right)$ by Lemma 2.1. By a standard diagonal process, we can find a subsequence $\left\{u_{n_{k}}\right\}$ of $\left\{u_{n}\right\}$ such that $\left\{L_{\alpha} u_{n_{k}}\right\}$ converges in $\left(\mathcal{K}\left(R^{p}\right), h\right)$ for each rational $\alpha \in[0,1]$.

Now it suffices to show that $\left\{u_{n_{k}}\right\}=\left\{v_{k}\right\}$ is a Cauchy sequence in $\left(\mathcal{F}\left(R^{p}\right), d_{\infty}\right)$. For a given $\varepsilon>0$, by (3.13) we can choose a $\delta>0$ such that

$$
\phi_{\infty}(u, \delta)<\varepsilon \text { for all } u \in \mathcal{U} .
$$

And then, we take a partition $0=\alpha_{0}<\alpha_{1}<\cdots<\alpha_{r}=$ 1 of $[0,1]$ satisfying $\alpha_{i}-\alpha_{i-1}<\delta$ for all $i$ and each $\alpha_{i}$ is rational.

Next we choose $k_{0}$ so that $m, k \geq k_{0}$ implies

$$
h\left(L_{\alpha_{i}} v_{m}, L_{\alpha_{i}} v_{k}\right)<\varepsilon \text { for all } i=0,1, \ldots, r \text {. }
$$

Now let $\alpha \in[0,1]$ and $\alpha \notin\left\{\alpha_{0}, \alpha_{1}, \ldots, \alpha_{r}\right\}$. Then there is an $\alpha_{i}$ so that $\alpha_{i}-\delta<\alpha<\alpha_{i}$ and so

$$
h\left(L_{\alpha} v_{k}, L_{\alpha_{i}} v_{k}\right)<\varepsilon \text { for all } k \text {. }
$$

Therefore, we obtain that for $m, k \geq k_{0}$,

$$
\begin{aligned}
& h\left(L_{\alpha} v_{m}, L_{\alpha} v_{k}\right) \\
\leq & h\left(L_{\alpha} v_{m}, L_{\alpha_{i}} v\right)+h\left(L_{\alpha_{i}} v_{m}, L_{\alpha_{i}} v_{k}\right)+h\left(L_{\alpha_{i}} v_{k}, L_{\alpha} v_{k}\right) \\
< & 3 \varepsilon
\end{aligned}
$$

which implies $d_{\infty}\left(v_{m}, v_{k}\right)<3 \varepsilon$. This completes the proof.

Corollary 3.9. Let $\left\{\tilde{X}_{n}\right\}$ be a sequence of fuzzy random variables. Then $\left\{\tilde{X}_{n}\right\}$ is strongly tight if

\section{1. $\left\{\left\|\tilde{X}_{n}\right\|\right\}$ is tight.}

2. For each $\varepsilon>0$ and $\eta>0$, there exists $a \delta \in(0,1)$ such that for all $n$,

$$
P\left(\phi_{\infty}\left(\tilde{X}_{n}, \delta\right) \geq \eta\right) \leq \varepsilon
$$

Finally, we wish to establish $L_{r}$-tightness of fuzzy random variables. To this end, we need a characterization of compact subsets of $\left(\mathcal{F}\left(R^{p}\right), d_{r}\right)$. This was firstly given by Diamond and Kloeden [4] in the space $F\left(R^{p}\right)$ of fuzzy convex subsets. Later on, Ming [18] modified the characterization of compact sets in [4]. Recently, Wu and Zhao [21] gave an counter-example which shows that the modified characterization in [18] is incorrect, and obtained correct characterization of compact sets in $\left(F\left(R^{p}\right), d_{r}\right)$. The techniques used in [21] is also available in $\left(\mathcal{F}\left(R^{p}\right), d_{r}\right)$.

For $u \in \mathcal{F}\left(R^{p}\right)$ and for $0<\delta<1$, let us denote

$$
\phi_{r}(u, \delta)=\int_{\delta}^{1} h\left(L_{\alpha} u, L_{\alpha-\delta} u\right)^{r} d \alpha .
$$

Theorem 3.10. Let $\mathcal{U}$ be a subset of $\left(\mathcal{F}\left(R^{p}\right), d_{r}\right)$. Then $\mathcal{U}$ is relatively compact if

(3.14) $\sup _{u \in \mathcal{U}}\|u\|<\infty$

(3.15) $\lim _{\delta \rightarrow 0} \sup _{u \in \mathcal{U}} \phi_{r}(u, \delta)=0$.

Proof. We show that every sequence in $\mathcal{U}$ has a convergent subsequence.

Let $\left\{u_{n}\right\}$ be a sequence in $\mathcal{U}$. Then (3.14) implies that for each $\alpha \in[0,1],\left\{L_{\alpha} u_{n}\right\}$ is relatively compact subset of $\left(\mathcal{K}\left(R^{p}\right), h\right)$ by Lemma 2.1. By a standard diagonal process, we can find a subsequence $\left\{u_{n_{k}}\right\}$ of $\left\{u_{n}\right\}$ such that $\left\{L_{\alpha} u_{n_{k}}\right\}$ converges in $\left(\mathcal{K}\left(R^{p}\right), h\right)$ for each $\alpha \in Q$, where $Q$ is the set of all rational points in $[0,1]$. Let

$$
A_{\alpha}=\left\{\begin{array}{lll}
\lim _{k \rightarrow \infty} L_{\alpha} u_{n_{k}} & \text { if } & \alpha \in Q, \\
\inf _{\beta \in Q, \beta<\alpha} A_{\beta} & \text { if } & \alpha \notin Q .
\end{array}\right.
$$

Then there exists an $u \in \mathcal{F}\left(R^{p}\right)$ such that $A_{\alpha}=L_{\alpha} u$ for all $\alpha \in[0,1]$. Now, (3.15) and the inequality

$$
\begin{aligned}
& \left(\int_{\delta}^{1} h\left(L_{\alpha} u_{n_{k}}, L_{\alpha} u\right)^{r} d \alpha\right)^{1 / r} \\
\leq \quad & \left(\int_{\delta}^{1} h\left(L_{\alpha} u_{n_{k}}, L_{\alpha-\delta} u_{n_{k}}\right)^{r} d \alpha\right)^{1 / r} \\
& +\left(\int_{\delta}^{1} h\left(L_{\alpha-\delta} u_{n_{k}}, L_{\alpha-\delta} u\right)^{r} d \alpha\right)^{1 / r} \\
& +\left(\int_{\delta}^{1} h\left(L_{\alpha-\delta} u, L_{\alpha} u\right)^{r} d \alpha\right)^{1 / r}
\end{aligned}
$$

implies that $d_{r}\left(u_{n_{k}}, u\right) \rightarrow 0$. This completes the proof.

Theorem 3.11. Let $\mathcal{U}$ be a subset of $\left(\mathcal{F}\left(R^{p}\right), d_{r}\right)$. Then $\mathcal{U}$ is relatively compact if and only if

$(3,16) \sup _{u \in \mathcal{U}} \int_{0}^{1}\left\|L_{\alpha} u\right\|^{r} d \alpha<\infty$.

(3.17) $\lim _{\delta \rightarrow 0} \sup _{u \in \mathcal{U}} \phi_{r}(u, \delta)=0$.

(3.18) Let $\left\{\alpha_{i}\right\}$ be a decreasing sequence in $(0,1]$ converging to 0 . For a sequence $\left\{u_{n}\right\}$ in $\mathcal{U}$, if $\left\{u_{n}^{\left(\alpha_{i}\right)}\right\}$ converges to $u_{\alpha_{i}} \in \mathcal{F}\left(R^{p}\right)$ in $d_{r}$, then there exists a $u_{0} \in$ $\mathcal{F}\left(R^{p}\right)$ such that $L_{\alpha} u_{0}=L_{\alpha} u_{\alpha_{i}}$ for all $\alpha_{i}<\alpha \leq 1$, where $u^{\left(\alpha_{i}\right)}=u I_{L_{\alpha_{i}} u}$, i.e.,

$$
u^{\left(\alpha_{i}\right)}(x)=\left\{\begin{array}{lll}
u(x) & \text { if } & u(x) \geq \alpha_{i} \\
0 & \text { if } & u(x)<\alpha_{i}
\end{array}\right.
$$


Proof. The proof is similar to that of Theorem 3.2 in Wu and Zhao [21].

The above Theorems 3.10 and 3.11 yield the followings.

Theorem 3.12. Let $\left\{\tilde{X}_{n}\right\}$ be a sequence of fuzzy random variables. Then $\left\{\tilde{X}_{n}\right\}$ is $L_{r}$-tight if

1. $\left\{\left\|\tilde{X}_{n}\right\|\right\}$ is tight,

2. For each $\varepsilon>0$ and $\eta>0$, there exists $a \delta \in(0,1)$ such that for all $n$,

$$
P\left(\phi_{r}\left(\tilde{X}_{n}, \delta\right) \geq \varepsilon\right) \leq \eta .
$$

Theorem 3.13. Let $\left\{\tilde{X}_{n}\right\}$ be a sequence of fuzzy random variables. If $\left\{\tilde{X}_{n}\right\}$ is $L_{r}$-tight, then

1. $\left\{\int_{0}^{1}\left\|L_{\alpha} \tilde{X}_{n}\right\|^{r} d \alpha\right\}$ is tight,

2. For each $\varepsilon>0$ and $\eta>0$, there exists $a \delta \in(0,1)$ such that for all $n$,

$$
P\left(\phi_{r}\left(\tilde{X}_{n}, \delta\right) \geq \varepsilon\right) \leq \eta
$$

\section{Counter-examples.}

From relations between several metrics on $\mathcal{F}\left(R^{p}\right)$ and Theorems 3.3 and 3.5, we can obtain the relationships between the concepts of tightness as follows;

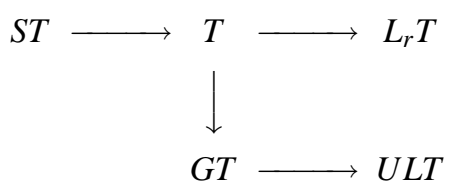

We give counter-examples which show that the inverse implications are not true.

Example 4.1. Let $\left\{u_{n}\right\}$ be a sequence in $\mathcal{F}\left(R^{p}\right)$ such that $\left\{u_{n}\right\}$ converges w.r.t. $d_{1}$ but any subsequence of $\left\{u_{n}\right\}$ does not converge w.r.t. $d_{s}$. Such a sequence exists(see Joo ad $\operatorname{Kim}[9])$. Then $\mathcal{U}=\left\{u_{n}: n=1,2, \ldots\right\}$ is compact in $\left(\mathcal{F}\left(R^{p}\right), d_{1}\right)$, but not in $\left(\mathcal{F}\left(R^{p}\right), d_{s}\right)$. Thus if we take $P\left(\tilde{X}_{n}=u_{n}\right)=1$, then $\left\{\tilde{X}_{n}\right\}$ is $L_{1}$-tight but not tight.

Similarly, if we choose a sequence $\left\{u_{n}\right\}$ in $\mathcal{F}\left(R^{p}\right)$ such that $\left\{u_{n}\right\}$ converges w.r.t. $D$ but any subsequence of $\left\{u_{n}\right\}$ does not converge w.r.t. $d_{s}$, (for such a sequence, see Example 3.1 of Kim and Kim [14]), we can give an counterexample that GT does not imply T. The next example shows that a single fuzzy random variable may not be strong tight.

Example 4.2. For $0<\lambda<1$, let

$$
u_{\lambda}(x)=\left\{\begin{array}{llc}
1, & \text { if } & x=0 \\
\lambda, & \text { if } & 0<|x| \leq 1 \\
0, & \text { elsewhere. } &
\end{array}\right.
$$

Then

$$
L_{\alpha} u_{\lambda}= \begin{cases}\{0\}, & \text { if } \lambda<\alpha \leq 1 \\ \{x:|x| \leq 1\}, & \text { if } 0 \leq \alpha \leq \lambda,\end{cases}
$$

and so $d_{\infty}\left(u_{\lambda}, u_{\delta}\right)=1$ for $\lambda \neq \delta$.

Now we let $\Omega=(0,1), \mathcal{A}=$ the Lebesque $\sigma$-field and $P$ be the Lebesgue measure. and define

$$
\tilde{X}: \Omega \rightarrow \mathcal{F}\left(R^{p}\right), \tilde{X}(\lambda)=\tilde{u}_{\lambda} .
$$

Then $\tilde{X}$ is a fuzzy random variable. Suppose that $0<\varepsilon<1$ and that there is a compact subset $\mathcal{U}$ of $\left(\mathcal{F}\left(R^{p}\right), d_{\infty}\right)$ such that

$$
P(\tilde{X} \notin \mathcal{U})<\varepsilon .
$$

Then $\mathcal{U}$ necessarily contains a set of the form

$$
\mathcal{U}_{J}=\left\{u_{\lambda}: \lambda \in J\right\}
$$

where $P(J)>1-\varepsilon$. But this is impossible because $\mathcal{U}_{J}$ contains a sequence $\left\{u_{\lambda_{n}}: \lambda_{n} \in J\right\}$ which does not have any convergent subsequence.

We recall that a single fuzzy random variable is tight because $\left(\mathcal{F}\left(R^{p}\right), d_{s}\right)$ is separable and topologically complete. Thus, the above example also shows that tightness does not imply strong tightness.

Example 4.3. Let us define

$$
u_{n}=\left\{\begin{array}{llc}
1 & \text { if } & x=0 \\
\frac{1}{n^{3}}(n-|x|) & \text { if } & 0<|x| \leq n \\
0 & \text { if } & |x|>n
\end{array}\right.
$$

Then

$$
L_{\alpha} u_{n}=\left\{\begin{array}{lll}
\{0\} & \text { if } & \frac{1}{n^{2}}<\alpha \leq 1 \\
\left\{x:|x| \leq n-n^{3} \alpha\right\} & \text { if } & 0 \leq \alpha \leq \frac{1}{n^{2}}
\end{array}\right.
$$

And then

$$
d_{1}\left(u_{n}, \tilde{0}\right)=\frac{1}{2 n} \rightarrow 0,
$$

and so $\mathcal{U}=\left\{u_{n}: n=1,2, \ldots\right\}$ is relatively compact subset of $\left(\mathcal{F}\left(R^{p}\right), d_{1}\right)$. Thus if $P\left(\tilde{X}_{n}=u_{n}\right)=1$, then $\left\{\tilde{X}_{n}\right\}$ is $L_{1}$ tight. But $\left\{\tilde{X}_{n}\right\}$ is not uniformly level-wise tight because $P\left(\left\|\tilde{X}_{n}\right\|=n\right)=1$. 
Example 4.4. Let us define

$$
u_{n}=\left\{\begin{array}{llc}
1 & \text { if } & x=0 \\
\frac{1-|x|}{n} & \text { if } & 0<|x| \leq 1 \\
0 & \text { if } & |x|>1
\end{array}\right.
$$

Then

$$
L_{\alpha} u_{n}=\left\{\begin{array}{lll}
\{0\} & \text { if } & \frac{1}{n}<\alpha \leq 1, \\
\{x:|x| \leq 1-n \alpha\} & \text { if } & 0 \leq \alpha \leq \frac{1}{n}
\end{array}\right.
$$

And so

$$
h\left(L_{0} u_{n}, L_{\alpha} u_{n}\right)=\left\{\begin{array}{lll}
1 & \text { if } & \frac{1}{n}<\alpha \leq 1 \\
n \alpha & \text { if } & 0 \leq \alpha \leq \frac{1}{n}
\end{array}\right.
$$

Hence $\sup _{n} \phi_{D}\left(u_{n}, \delta\right)=1$ for all $\delta>0$. This implies that $\mathcal{U}=\left\{u_{n}: n=1,2, \ldots\right\}$ is not relatively compact in $\left(\mathcal{F}\left(R^{p}\right), D\right)$ i.e., any subsequence of $\left\{u_{n}\right\}$ does not converge. If we take $P\left(\tilde{X}_{n}=u_{n}\right)=1$, then $\left\{\tilde{X}_{n}\right\}$ is not GT But $\left\{\tilde{X}_{n}\right\}$ is ULT because $\left\|\tilde{X}_{n}\right\|=1$ for all $\mathrm{n}$.

\section{References}

[1] P. Billingsley, Convergence of probability measures, Second Edition, Wiley, New York, 1999.

[2] A. Colubi, J. S. Domingeuz-Menchero, M. LopezDiaz and D. Ralescu, "A $D_{E}[0,1]$ representation of random upper semicontinuous functions", Proc. Amer. Math. Soc. vol. 130, pp. 3237-3242, 2002.

[3] G. Debreu, "Integration of correspondences", Proc. 5th Berkeley Symp. Math. Statist. Prob. vol. 2, pp. 351-372, 1966.

[4] P. Diamond and P. Kloeden, "Metric space of fuzzy sets", Fuzzy Sets and Systems vol. 35, pp. 241-249, 1990.

[5] P. Diamond and P. Kloeden, Metric spaces of fuzzy sets: Theory and Applications, World Scientific, Singapore, 1994.

[6] T. Fan, "On the compactness of fuzzy numbers with sendograph metric", Fuzzy Sets and Systems vol. 143, pp. 471-477, 2004.

[7] G. H. Greco, "Sendograph metric and relatively compact sets of fuzzy sets", Fuzzy Sets and Systems vol. 157, pp. 286-291, 2006

[8] G. H. Greco and M. P. Moschen, "Supremum metric and relatively compact sets of fuzzy sets", Nonlinear Anal. vol. 64, pp. 1325-1335, 2006.

[9] H. Inoue, "A strong law of large numbers for fuzzy random sets", Fuzzy Sets and Systems vol. 41, pp. 285-291, 1991.
[10] S. Y. Joo and Y. K. Kim, "Topological properties on the space of fuzzy sets", J. Math. Anal. Appl. vol. 246, pp. 576-590, 2000.

[11] S. Y. Joo and Y. K. Kim, "Weak convergence and tightness for fuzzy random variables", Indian J. Pure Appl. Math. vol. 35, pp. 793-809, 2004.

[12] S. Y. Joo, G. S. Choi, J. S. Kwon and Y. K. Kim, "Some results on convergence in distribution for fuzzy random sets", J. Korean Math. Soc. vol. 42, pp. 171-189, 2005.

[13] S. Y. Joo, Y. K. Kim, J. S. Kwon and G. S. Choi, "Convergence in distribution for level-continuous fuzzy random sets", Fuzzy Sets and Systems vol. 157, pp. 243-255, 2006.

[14] D.S. Kim and Y. K. Kim, "Some properties of a new metric on the space of fuzzy numbers", Fuzzy Sets and Systems vol. 145, pp. 395-410, 2004.

[15] Y. K. Kim, "Measurability for fuzzy valued functions", Fuzzy Sets and Systems vol. 129, pp. 105-109, 2002.

[16] E. P. Klement, M. L. Puri and D. A. Ralescu, "Limit theorems for fuzzy random variables", Proc. Roy. Soc. Lond. Ser. A vol.407, pp. 171-182, 1986.

[17] S. Li, Y. Ogura and V. Kreinovich, Limit Theorems and Applications of Set-valued and Fuzzy Setvalued Random Variables, Kluwer Academic Publishers, Dordrecht, 2002.

[18] M. Ming, "Some notes on the characterization of compact sets in $\left(E^{n}, d_{p}\right)$ ', Fuzzy Sets and Systems vol. 56, pp. 297-301, 1993.

[19] Yu. V. Prokhorov, "Convergence of random processes and limit theorems in probability theory", Theory Probab. Appl. vol. 1, pp. 157-214, 1956.

[20] M. L. Puri and D. A. Ralescu, "Fuzzy random variables", J. Math. Anal. Appl. vol. 114, pp. 402-422, 1986.

[21] C.Wu and Z. Zhao, "Some notes on the characterization of compact sets of fuzzy sets with $L_{p}$ metric", Fuzzy Sets and Systems vol. 159, pp. 2104-2115, 2008.

\section{Yun Kyong Kim}

Professor of Dongshin University

Research Area: Fuzzy Probability Theory, Fuzzy Analysis and Related Fields

E-mail : ykkim@dsu.ac.kr 